\title{
HÁBITOS DE ALIMENTACIÓN ASOCIADOS A SOBREPESO Y OBESIDAD EN ADULTOS MEXICANOS: UNA REVISIÓN INTEGRATIVA
}

\author{
EATING HABITS ASSOCIATED WITH OVERWEIGHT AND OBESITY IN \\ MEXICAN ADULTS: AN INTEGRATIVE REVIEW
}

\section{HÁBITOS ALIMENTARES ASSOCIADOS AO SOBREPESO E OBESIDADE EM ADULTOS MEXICANOS: UMA REVISÃO INTEGRATIVA}

\author{
Diana Mercedes Hernández-Corona* \\ MARIO ÁNGEL-GONZÁLEZ** \\ Julio CÉsAr VÁzQuez-Colunga*** \\ Andrea Berenice Lima-Colunga**** \\ Claudia Liliana VÁZQueZ-Juárez***** \\ Cecilia Colunga-Rodríguez ${ }^{* * * * * *}$
}

\begin{abstract}
RESUMEN
Objetivo: Describir hallazgos publicados de hábitos de alimentación y su relación con sobrepeso y obesidad en adultos mexicanos. Material y Método: Revisión integrativa basada en 5 pasos sugeridos por Crossetti, utilizando las palabras clave "hábitos de alimentación", "sobrepeso", "obesidad" y "adultos mexicanos", con operadores booleanos "AND” y "OR"; se buscó en Scielo, PubMed, Conricyt, Scopus y WOS, entre los años 2005 y 2020, publicaciones en español e inglés. Resultados: Se identificaron 64 artículos, de estos se descartaron 52, quedando 12 acordes a criterios de inclusión; los artículos fueron analizados en extenso, identificando 4 categorías: 1) Zona de vivienda; 2) Estrato socioeconómico; 3) Género y 4) Cultura; cada una de ellas con subcategorías. Conclusiones: Las zonas rurales reportan mayor consumo de frutas, verduras, cereales, vitaminas y menor consumo calórico; en zonas urbanas se consume mayor cantidad de grasa, alimentos procesados, azúcares refinadas y menos vegetales, frutas y cereales. Un mayor estrato socioeconómico se asocia con alimentación menos calórica y comidas más naturales. Los hombres consumen más carne, grasa y alcohol, las mujeres
\end{abstract}

\footnotetext{
*Doctora en Farmacología. Centro Universitario de Tonalá, Universidad de Guadalajara, México. ORCID: https://orcid. org/0000-0002-8631-9201 Email: diana.hcorona@academicos.udg.mx

** Doctor en Psicología. Centro Universitario de Tonalá, Universidad de Guadalajara, México. ORCID: https://orcid.org/00000002-5762-5170 Email: mario.angel@academicos.udg.mx

*** Doctor en Psicología. Centro Universitario de Tonalá, Universidad de Guadalajara, México. ORCID: https://orcid.org/00000001-8393-2505 Email: julio.vazquez@academico.udg.mx Autor de correspondencia.

**** Maestra en Ciencias de la Salud en el Trabajo. Centro Universitario de Ciencias de la Salud, Universidad de Guadalajara, México. ORCID: https://orcid.org/0000-000-0003-4627-1757 Email: ln.andreablc@gmail.com

******Doctora en Psicología. Centro Universitario de Tonalá, Universidad de Guadalajara, México. ORCID: https://orcid. org/0000-0002-0932-0110 Email: claudia.vazquez5425@academicos.udg.mx Autor de correspondencia.

****** Doctora en Ciencias de la Salud Pública. Hospital de Pediatría Centro Médico Nacional de Occidente, Instituto Mexicano del Seguro Social / Centro Universitario de Ciencias de la Salud, Universidad de Guadalajara, México. ORCID: https://orcid. org/0000-0003-0328-788X Email: cecilia.colunga@imss.gob.mx
} 
consumen más hidratos de carbono y azúcares refinadas. En cuestiones culturales reportan distracciones a la hora de comer, como uso de celular, bajo consumo de agua y alto consumo de gaseosas (70\%); refieren barreras para alimentarse sanamente, como el trabajo pagado y doméstico, realizando comidas a prisa, sin considerar la calidad de los alimentos que se consumen. Estos hallazgos sugieren considerar factores socioeconómicos y culturales en las acciones preventivas que los profesionales de enfermería realizan en la práctica clínica y comunitaria.

Palabras clave: Hábitos de alimentación; Sobrepeso; Obesidad; Adultos mexicanos.

\begin{abstract}
Objective: To describe published findings on eating habits and their relationship with overweight and obesity in Mexican adults. Materials and Methods: Integrative review based on 5 steps suggested by Crossetti, using the keywords "feeding habits", "overweight", "obesity" and "Mexican adults" along with Boolean operators "AND" and "OR" in the Scielo, PubMed, Conricyt, Scopus and WOS databases, with publications in Spanish and English, between the years 2005 and 2020. Results: 64 articles were identified and 52 were discarded, leaving 12 that complied with the inclusion criteria. The articles were extensively analyzed, identifying 4 categories: 1 ) Housing area; 2) Socioeconomic stratum; 3) Gender and 4) Culture; each of them divided into subcategories. Conclusions: Rural areas report higher consumption of fruits, vegetables, cereals, vitamins and lower caloric consumption, while in urban areas more fat, processed food, refined sugars and fewer vegetables, fruits and cereals are consumed. A higher socioeconomic stratum is associated with a less caloric diet and more natural food. Men consume more meat, fat and alcohol, while women consume more carbohydrates and refined sugars. Regarding cultural issues, people report distractions at mealtime, such as cell phone use, low water consumption and high consumption of soft drinks (70\%); they also refer to barriers to healthy eating, such as work, eating meals quickly without considering the quality of food. These findings suggest considering socioeconomic and cultural factors in the preventive actions that nursing professionals carry out in clinical and community practice.
\end{abstract}

Key words: Eating habits; Overweight; Obesity; Mexican adults.

\title{
RESUMO
}

Objetivo: Descrever os resultados publicados sobre hábitos alimentares e sua relação com sobrepeso e obesidade em adultos mexicanos. Material e Método: Revisão integrativa baseada em 5 etapas sugeridas por Crossetti, utilizando as palavras-chave "hábitos alimentares", "sobrepeso", "obesidade" e "adultos mexicanos", com operadores booleanos "AND” e "OR", a busca foi realizada em Scielo, PubMed, Conricyt, Scopus e WOS, entre os anos de 2005 e 2020, publicações em espanhol e inglês. Resultados: Foram identificados 64 artigos, destes, 52 foram descartados, restando 12, de acordo com os critérios de inclusão; os artigos foram amplamente analisados, identificando 4 categorias: 1) Área de habitaçáo; 2) Estrato socioeconômico; 3) Gênero e, 4) Cultura; cada um deles com subcategorias. Conclusóes: As áreas rurais relatam maior consumo de frutas, hortaliças, cereais, vitaminas e menor consumo calórico; nas áreas urbanas, o consumo maior é de gordura, alimentos processados, açúcares refinados e menor consumo de vegetais, frutas e cereais. Um estrato socioeconômico mais elevado está associado a uma dieta menos calórica e alimentos mais naturais. Os homens consomem mais carne, gordura e álcool, as mulheres consomem mais carboidratos e açúcares refinados. Em questôes culturais, relatam distraçôes na hora das refeiçôes, como uso do celular, baixo consumo de água e alto consumo de refrigerantes $(70 \%)$, referem-se a barreiras para uma alimentaçáo saudável, como trabalho remunerado e doméstico, alimentação rápida, sem considerar a qualidade dos alimentos consumidos. Os resultados sugerem considerar fatores socioeconômicos e culturais nas açóes preventivas que os profissionais de enfermagem realizam na prática clínica e comunitária.

Palavras-chave: Hábitos alimentares; Sobrepeso; Obesidade; Adultos mexicanos.

Fecha de recepción: 24/01/2020

Fecha de aceptación: 28/12/2020 


\section{INTRODUCCIÓN}

En el mundo hay más de 1.900 millones de adultos con sobrepeso, de los cuales 650 millones presentan obesidad $^{(1)}$. México ocupa el segundo lugar a nivel mundial en obesidad en población adulta, lo cual es 10 veces mayor que en países como Japón y $\mathrm{Corea}^{(2)}$, asimismo, es primer lugar en América Latina ${ }^{(3)}$. En población mexicana de 20 o más ańos de edad, la prevalencia combinada de sobrepeso y obesidad pasó de $71,3 \%$ en el año 2012, a 75,2\% en $2018^{(1)}$, además, la prevalencia de obesidad abdominal es de $76,6 \%{ }^{(4)}$. Esto es un grave problema de salud pública, no solo por ser una pandemia ${ }^{(5)}$, sino porque la obesidad predispone a enfermedades crónicas graves, que son las primeras causas de muerte en mexicanos, como la hipertensión arterial y la diabetes mellitus tipo $2^{(4)}$, además, recientemente se ha documentado que la obesidad acarrea mayor riesgo de infección, hospitalización, gravedad y muerte por COVID-19, respecto a las personas con normopeso ${ }^{(5,6)}$.

En el año 2018, la Encuesta Nacional de Salud y Nutrición (ENSANUT) de México reportó que el sobrepeso y la obesidad en adultos, según el sexo, llegó a un 73\% en hombres y un 76,8\% en mujeres ${ }^{(7)}$. Este problema surge desde la niñez por los hábitos alimentarios, que en la edad adulta son difíciles de modificar, debido a costumbres y rutina; asimismo, se han reconocido componentes adictivos en alimentos que contienen azúcares y grasas, sobre todo los de bajo aporte nutricional, cuyo exceso genera enfermedades metabólicas crónicas $^{(4,8)}$.

Los hábitos de alimentación son definidos como aquellas acciones por las cuales elegimos lo que comemos, llevándonos a la selección, preparación e ingesta; son comportamientos individuales y grupales, resultantes de prácticas socioculturales ${ }^{(2)}$. Por otra parte, la alimentación es un proceso voluntario y social, a través del cual se aportan nutrientes y energía para las funciones físicas y los procesos metabólicos ${ }^{(4,9)}$.

En las últimas décadas se ha observado un cambio drástico en la alimentación de los mexicanos $^{(10,11)}$; atribuyéndose a la influencia de factores económicos, sociales y familiares, así como al crecimiento demográfico y horarios laborales y académicos los que provocan limitaciones para acceder fácilmente a la adquisición y mantenimiento de hábitos alimentarios saludables. Asimismo, aspectos como las tradiciones culturales, han inculcado el consumo de alimentos altos en grasas saturadas y azúcares refinadas, así como productos no saludables ${ }^{(2)}$.

Las evidencias revelan que los malos hábitos alimentarios son un factor relevante en el aumento de peso y la consecuente sobrecarga de volumen, hipertensión, desregulación metabólica, activación neurohumoral e inflamación sistémica ${ }^{(5)}$. La obesidad es considerada una enfermedad multifactorial, condicionada por factores genéticos psicológicos y ambientales ${ }^{(5,2)}$ y se estima que al menos 7 de cada 10 mexicanos adultos tienen sobrepeso u obesidad ${ }^{(8)}$. Estas alarmantes cifras sugieren la urgente necesidad de adoptar hábitos alimentarios adecuados y acciones como aumentar la actividad física, la selección de alimentos sanos y el manejo del estrés, evitando complicaciones en la población y que mejoren su calidad de vida ${ }^{(12)}$; lo cual se considera no sólo un indicador de desarrollo personal, sino que constituye un indicador social y económico a nivel nacional ${ }^{(13)}$.

El personal de enfermería y otros profesionales de la salud se enfrentan, día a día, a la atención y cuidado de pacientes con hábitos poco saludables, debiendo realizar acciones preventivas, de educación y promoción de la salud, tanto a nivel clínico como en el ámbito comunitario desde la salud pública; de ahí que los resultados de esta revisión pueden ser de utilidad para esta importante labor. Por lo anterior, el objetivo de este trabajo fue identificar los hábitos de alimentación en adultos mexicanos, asociados al sobrepeso y obesidad descritos en la literatura científica en los últimos 15 años.

\section{MATERIAL Y MÉTODO}

Se realizó una revisión integrativa, siguiendo las cinco etapas sugeridas por Crossetti $\left.{ }^{(14)}: 1\right)$ Delimitación del problema y formulación de la pregunta de investigación; 2) Búsqueda de literatura, acorde a los criterios establecidos; 3) Evaluación de los artículos; 4) Síntesis de los artículos y 5) Interpretación de los resultados identificados en las publicaciones. La búsqueda se llevó a cabo en las bases de datos de Scielo, PubMed, Conricyt, Scopus y Web of Science (WOS), usando las palabras clave: hábitos de alimentación, sobrepeso, obesidad y adultos 
mexicanos, con su traducción respectiva en inglés (feeding habits, overweight, obesity and mexican adults) y vinculadas con los operadores booleanos "AND" y "OR". Entre los criterios de inclusión de los documentos, se consideró que fueran artículos científicos publicados en el periodo comprendido entre los año 2005 al 2020, disponibles en línea, con acceso abierto y en el idioma inglés o español; fueron excluidos documentos correspondientes a tesis, libros, editoriales, capítulos, artículos de revisión y ensayos académicos.

Se encontraron 64 artículos y todos fueron analizados en extenso por 3 revisores en modalidad cegada, coincidiendo por unanimidad en la selección de los incluidos a excepción de 2, que pasaron a un cuarto revisor; se descartaron un total de 52. Los motivos para no incluir los artículos fueron porque 14 de ellos estaban relacionados con enfermedades crónicas como diabetes, hipertensión, cáncer y problemas gastrointestinales, 9 por contener información relacionada con hábitos de alimentación en etapa escolar, adolescencia o en adultos mayores, 7 por incluir mujeres en lactancia o maternidad o por referirse a cirugía bariátrica, 4 por tratarse de poblaciones de otras nacionalidades, 4 se referían a intervenciones para disminuir la obesidad, 1 se trataba de tesis de grado, 3 se referían a poblaciones de trabajadores vinculadas con su actividad laboral, 1 se refería a aspectos moleculares de la nutrición, 3 no tenían acceso abierto, 3 estaban duplicados y 1 por tratarse de la validación de un test que no presentó datos respecto a los hábitos de alimentación de riesgo para obesidad. Los 2 artículos que fueron revisados por un cuarto revisor, quien fungió como árbitro, no fueron incluidos, por no ajustarse cabalmente a los criterios de selección y calidad. Finalmente se eligieron 12 artículos para la revisión integrativa y fueron analizados para extraer categorías conceptuales de afinidad y diferencias. La decantación de los documentos, según la base de datos de donde fueron extraídos, se muestra en la Figura 1.

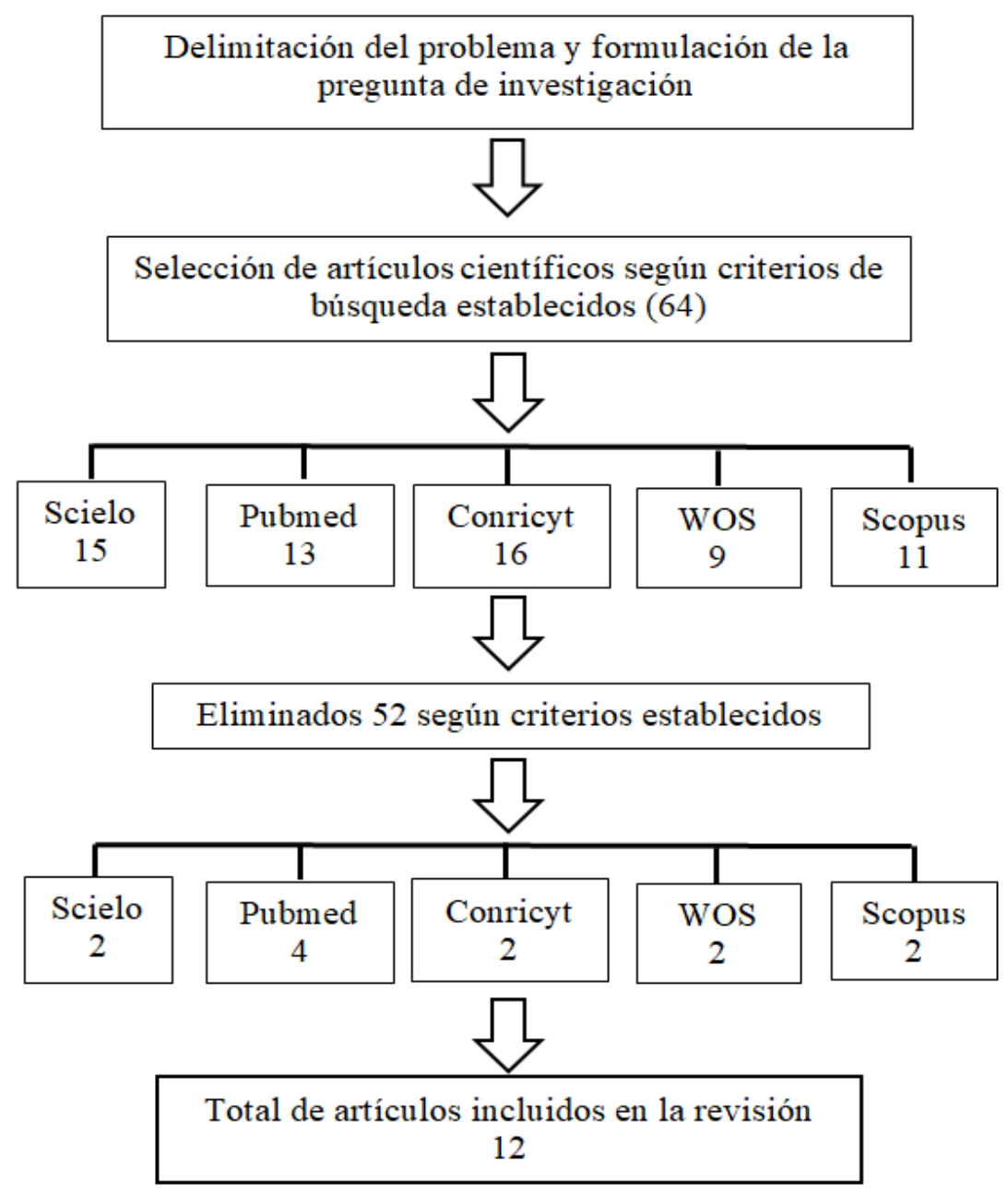

Figura 1. Procedimiento de selección de artículos, período 2005-2020. 


\section{RESULTADOS}

De los 12 artículos, 4 fueron publicados en inglés, 4 en los últimos 5 años, 2 con diseños cualitativos por medio de entrevistas; 10 cuantitativos, con diseños transversales, cohorte y descriptivos, usando cuestionarios, recordatorio de 24 horas y 7 días, inventarios y pruebas estandarizadas tipo test. Abarcaron 10.332 adultos con edades mayores de 20 años, de los cuales, 3.511 fueron hombres $(33,98 \%)$ y 6.821 mujeres $(66,02 \%)$. En la Tabla 1 se señalan los principales datos. Partiendo de la variable central "Hábitos de alimentación", seguida de las prevalencias de sobrepeso y obesidad, se identificaron cuatro categorías: 1) Zona de vivienda; 2) Estrato socioeconómico; 3) Género y 4) Cultura; a su vez, cada una de las categorías se subdividió en subcategorías (Figura 2).

1. Zona de vivienda, lo urbano y lo rural: $\mathrm{La}$ zona de vivienda se refiere a la ubicación geográfica y a las características del ambiente donde habitan las personas, que puede presentar cierto grado de desarrollo e industrialización, para este caso, zona urbana o zona $\operatorname{rural}^{(9,15,16)}$. La primera se refiere a la vida en las ciudades altamente desarrolladas, con estilos de vida más sedentarios y consumo industrializado ${ }^{(9)}$. Los resultados de la revisión muestran patrones de consumo asociados al desarrollo socioeconómico y la urbanización ${ }^{(9,15,16)}$. Las zonas urbanas presentan mayor consumo de nutrimentos ${ }^{(15)}$, con una disminución en el consumo de carnes, pescado, huevo, frutas y verduras, con un aumento en el consumo de cereales, tubérculos, aceites, grasas, refrescos y alimentos procesados, con altos contenidos de conservadores ${ }^{(9,15)}$.

En las zonas rurales se presenta un consumo energético menor y menos ingesta de grasas, no obstante, un consumo alto de vitamina $\mathrm{C}$ y cereales $^{(15)}$. Los hallazgos sugieren que en las zonas urbanas, con mayor desarrollo, aunque se consumen mayor variedad de nutrimentos ${ }^{(15,16)}$, también se consume mayores cantidades de calorías, vitaminas, cereales integrales, grasa y azúcares refinadas, en comparación con las zonas rurales ${ }^{(15)}$. No obstante las diferencias estadísticas en los hábitos de consumo entre zonas, llama la atención que el problema del sobrepeso y la obesidad en adultos es alto tanto en zonas rurales como urbanas en México, lo cual se puede atribuir a que en las zonas rurales ha ido en aumento el consumo de alimentos procesados y refrescos ${ }^{(15,17,18)}$.

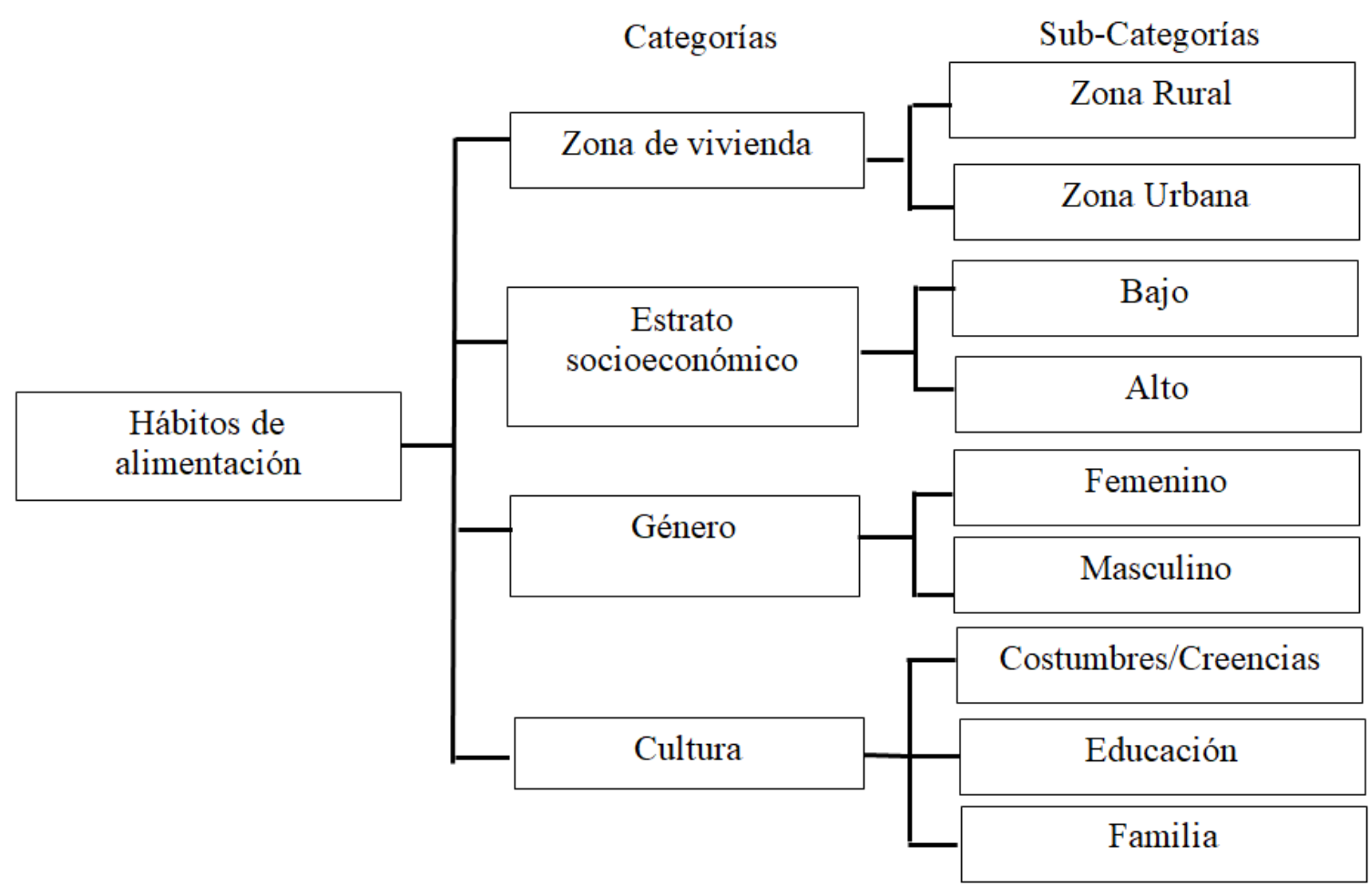

Figura 2. Categorías y subcategorías extraídas de los artículos. 


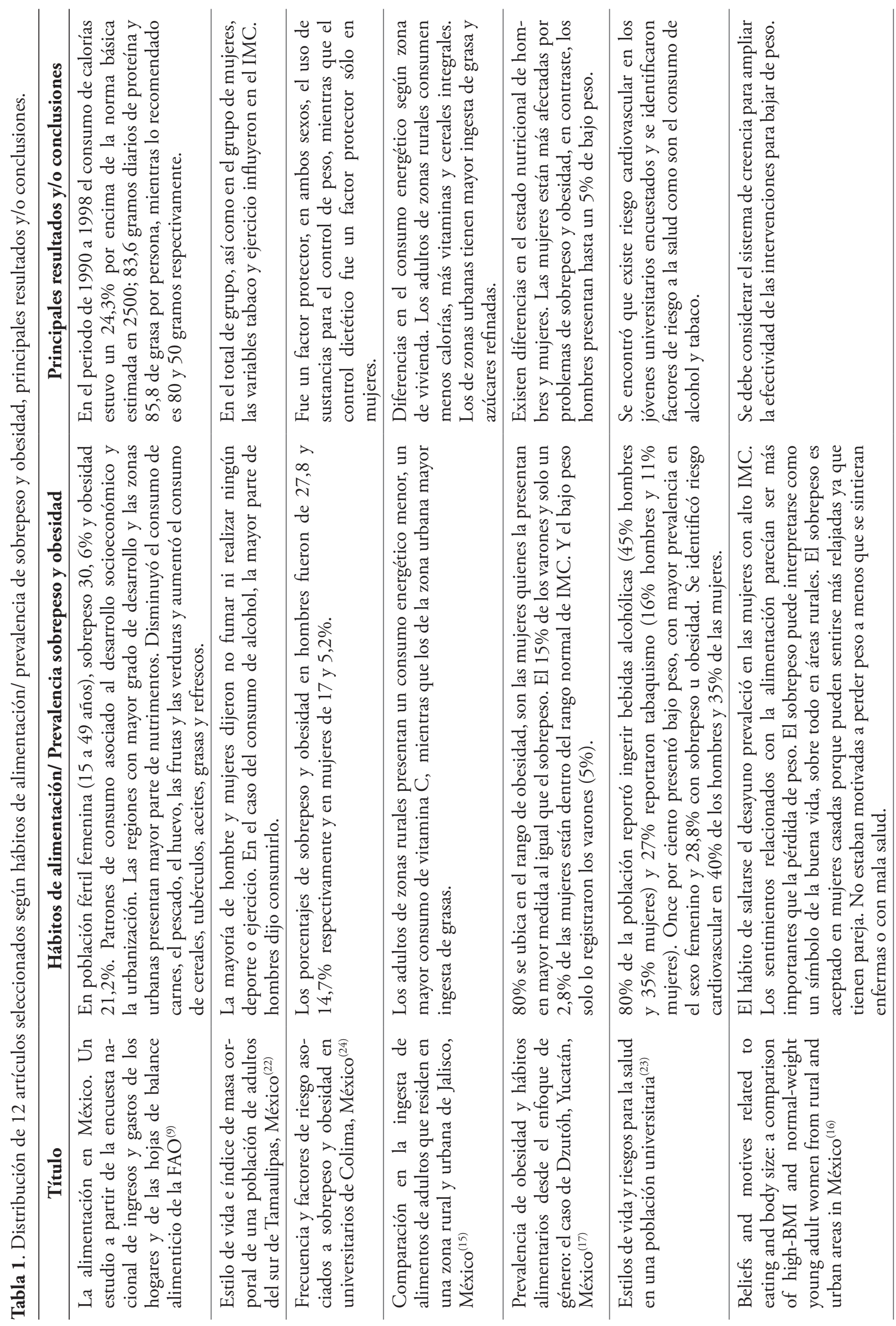




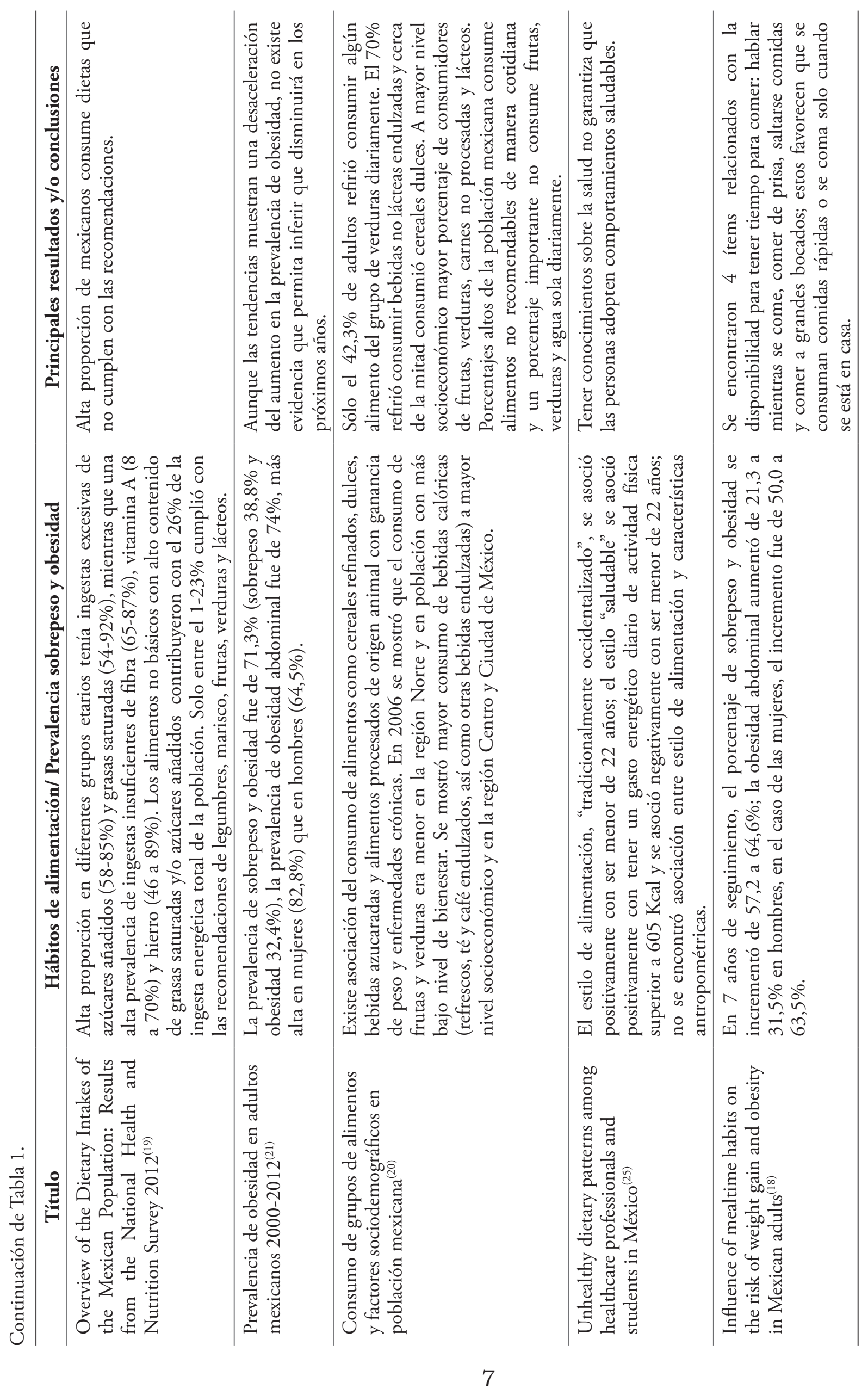




\section{Estrato socioeconómico bajo y alto: $\mathrm{El}$} estrato socioeconómico se refiere básicamente a características como ingresos monetarios y nivel de bienestar de la vivienda, entre otros indicadores ${ }^{(9)}$. Se puede observar que en la población de estratos socioeconómicos bajos, las personas consumen menor cantidad de frutas y verduras respecto a los adultos de estratos más altos ${ }^{(9)}$, donde se presentan patrones de consumo con mayor cantidad de bebidas calóricas como refrescos, té y café endulzados ${ }^{(19,20)}$.

Así, la malnutrición se considera como un indicador que describe las diferencias sociales y económicas que impactan el estado de salud de las poblaciones ${ }^{(9)}$. La falta de recursos e incertidumbre económica para la planificación de un menú, así como la falta de conocimientos nutricionales, desencadenan en malas prácticas alimentarias. Esto es característico de zonas geográficas con bajo desarrollo económico. En México existen diferencias económicas y por ende, en el consumo de alimentos y sobrepeso, teniendo algunos estados del norte más recursos económicos, pero más afectación, por ejemplo, Baja California sur tiene la mayor prevalencia de obesidad (80\%), contrario a Chiapas con $64,3 \%$, que se ubica al sureste del país y muestra menor desarrollo económico. Hacia el centro de México, se muestra mayor consumo de frutas y verduras, aunque la obesidad sigue estando por arriba del promedio en el país (74\%), sin embargo, a pesar de las diferencias, en México de manera general, se encuentra un alto consumo de refrescos y bebidas azucaradas y una cantidad insuficiente de agua sola, así como una grave prevalencia de obesidad $^{(9,20,21)}$.

3. Género femenino y masculino: El género es un factor relevante en los hábitos de alimentación del sobrepeso y la obesidad. Dadas sus grandes diferencias, tanto en consumo, prevalencia y la edad en que se presentan, son las mujeres las más afectadas a edades más tempranas, por lo que el enfoque de género se debe integrar a los nuevos estudios y programas de alimentación ${ }^{(17)}$. En la Encuesta Nacional de Salud y Nutrición de México, realizada el año 2012, aplicada a 38.208 mexicanos, se encontró que la prevalencia de obesidad abdominal fue de $82,8 \%$ en mujeres, mientras que en hombres fue de $62,5 \%$ y que la prevalencia de sobrepeso y obesidad fue mayor en las mujeres (73\%), que en los hombres $(69,4 \%)$ (17). Consistente con estos datos, en un estudio actual con seguimiento a 7 años, se reporta que la población femenina incrementó la obesidad abdominal de 50 a $63,5 \%$, lo que fue mayor que la presentada por los hombres en el mismo estudio $(21,3 \text { a } 31,5 \%)^{(18)}$.

En el estudio realizado en Tamaulipas, México, se observó que los hombres refieren mayor consumo de alcohol que las mujeres, la mayoría refiere no realizar ejercicio ni fumar; el ejercicio y el fumar mostraron relación negativa con el $\mathrm{IMC}^{(22)}$. Mientras, en un estudio realizado en una zona rural de Yucatán, al sureste de México, se encontró que el $80 \%$ de adultos se situaba en el rango de obesidad, además sólo el $15 \%$ de los hombres y el $2,8 \%$ de las mujeres presentaban IMC en rango de normalidad ${ }^{(20)}$.

En contraste, en estudiantes de ciencias biológicas de Guadalajara, de acuerdo a reportes de Gómez et al. $(2016)^{(23)}$, 60,1\% presentó peso normal, mayor prevalencia de obesidad en hombres $(21,6 \%)$ que en mujeres, pues sólo 7,2\% presentó sobrepeso u obesidad, aunque en ambos sexos se identificó riesgo cardiovascular, según su circunferencia de cintura ${ }^{(23)}$. También en población universitaria de Colima se identificó que el control dietético era un factor protector para la obesidad en mujeres, no así en los hombres ${ }^{(24)}$. Sin embargo, en ambas poblaciones de adultos universitarios se encontraron conductas de riesgo como fumar y beber alcohol con estrategias poco saludables para controlar el peso ${ }^{(23,24)}$.

4. Cultura, costumbres/creencias, educación, familia: Como parte de la cultura, las costumbres y creencias son factores que pueden ser determinantes de los hábitos de alimentación. En el estudio realizado por Caamaño et al. ${ }^{(16)}$, las mujeres refieren costumbres como saltarse las comidas, o realizar sólo dos comidas al día, debido a que así las habían acostumbrado desde pequeñas para ahorrar recursos, sin embargo, refieren un alto consumo de refresco de cola, el cual, incluso, lo consideran una adicción ${ }^{(16)}$. En cuanto a las creencias, en el mismo estudio, reconocen el aumento de peso en las mujeres casadas y en los niños, como signo de "buena vida" y de tener un esposo que es buen proveedor. Así las mujeres con sobrepeso casadas refieren sentirse más relajadas por ya tener pareja y debido a que consideran que son más importantes los sentimientos relacionados con la alimentación que bajar de peso ${ }^{(16,17)}$. 
Por su parte, la educación ha sido centro de interés de intervenciones para bajar de peso, no obstante, los esfuerzos no han rendido frutos frente a la publicidad comercial de las grandes empresas, que promueven estilos de vida y de alimentación poco saludables ${ }^{(17)}$. Ejemplos de que no han sido efectivas las estrategias educativas para la sana alimentación que se promueve en la escuela, son algunos resultados reportados en un estudio realizado en estudiantes universitarios en Colima, México, donde se encontró uso de sustancias para el control de peso, lo cual se comportó estadísticamente como factor protector ${ }^{(24)}$. Datos similares se hallaron en estudiantes universitarios de ciencias biológicas y ciencias de la salud (medicina, enfermería, nutrición, odontología, cultura física y deportes), donde se reportan hábitos de alimentación poco sanos, y en menores de 22 años se encontró un estilo de alimentación "occidentalizado"(23, 25), lo cual sugiere que la educación y los conocimientos, no aseguran hábitos saludables ${ }^{(25)}$.

Asimismo, la familia además de educar, reproduce la cultura dominante, configurándose en un factor fundamental de influencia acerca de las conductas de alimentación, además de ejercer presión sobre lo que debe ser la forma del cuerpo de los miembros de la familia, por ejemplo, en una mujer casada ${ }^{(16)}$. Existen datos reportados en mujeres de Querétaro, en la zona centro de México, donde las entrevistadas refirieron no estar motivadas a cambiar su alimentación para bajar de peso, a menos de que se sintieran enfermas o con mala salud $^{(19)}$, ya que el bajar de peso, siendo casadas, se puede interpretar como pretender gustarle a otros hombres, lo cual es socialmente desaprobado.

\section{DISCUSIÓN}

Se encontraron hábitos que no son compatibles con un peso saludable y que podrían explicar la gran problemática de obesidad que tiene México ${ }^{(1)}$.

Respecto a la zona de vivienda, los adultos de zonas urbanas consumen alimentos altos en grasa, procesados y azúcares refinadas. En la zona rural aún es mayor el consumo de alimentos naturales, sin embargo, hay alto consumo de refrescos embotellados de cola ${ }^{(4)} \mathrm{y}$ un progresivo incremento en consumo de comida procesada, botanas y comida rápida ${ }^{(11)}$. Un estudio en Nuevo León ${ }^{(26)}$ reportó un consumo calórico semanal similar en las zonas urbana y rural, lo cual es consistente con los hallazgos en esta revisión, además reporta similitud en obesidad y sobrepeso, agravado por el confinamiento por pandemia, por menor gasto calórico $^{(27)}$. Por otra parte, tener antecedentes de al menos un familiar directo con obesidad, no se relacionó con estar obeso, sin embargo, tener antecedentes familiares de obesidad y un consumo elevado de calorías, sí se relaciona ${ }^{(26)}$, lo cual sugiere la alta carga social del fenómeno y su carácter transdisciplinario.

Se observa que en la categoría Género existen importantes diferencias de hábitos de alimentación y obesidad entre hombres y mujeres ${ }^{(8)}$, lo que coincide con un estudio chileno, donde se estimó mayor riesgo de obesidad en mujeres adultas de 19 a 64 años, en comparación con sus pares masculinos; el problema es todavía mayor en mujeres de nivel socioeconómico medio y medio bajo ${ }^{(28)}$. Las mujeres refieren saltarse el desayuno y tienen más sobrepeso ${ }^{(16)}$. Esto concuerda con hallazgos en población japonesa donde los más obesos también se "saltan" una comida ${ }^{(29)}$; las mujeres refieren factores psicosociales y emocionales con la alimentación, que predominan sobre la pérdida de peso entre las de mayor $\mathrm{IMC}^{(16)}$. Destacan ideas del sobrepeso como símbolo de "buena vida", de tener un esposo proveedor y que hay mayor aceptación de la obesidad en mujeres casadas y niños ${ }^{(16)}$. Coincidentemente, el problema de sobrepeso y obesidad en México, es más grave en las mujeres y en los niños, teniendo el segundo y primer lugar mundial respectivamente ${ }^{(1)}$, lo cual sugiere un vínculo social entre el género y la crianza.

En los estratos socioeconómicos más bajos, las personas parecen estar más ansiosas por aprovechar buenos momentos como disfrutar de la comida, y dado que su futuro puede parecerles desafortunado, buscan experimentar una "buena vida” en el presente inmediato. En este estudio, las mujeres con alto IMC también parecían centrarse en disfrutar de la vida a corto plazo en lugar de evitar las consecuencias para su salud y, con ello, garantizar una vida feliz a largo plazo; entonces estar obesa es un símbolo de disfrutar de una buena $v i^{(16)}$. La mayoría de las mujeres con obesidad, revelaron sentirse orgullosas de comer en exceso, posiblemente para demostrar que tienen un mejor nivel socioeconómico o tener un mejor marido que 
otras mujeres $^{(16)}$.

Respecto a la categoría Cultura, Matus et al. ${ }^{(30)}$ refiere que hay factores socioculturales involucrados en el control de peso de la población adulta en Chiapas, México, tales como la autopercepción de la figura corporal, la subestimación del exceso de peso y del riesgo para la salud, el control de alimentos por cuestiones emocionales y la importancia de la influencia familiar, entre otros. Se ha encontrado un consumo de grandes cantidades de refresco, particularmente de refrescos de cola, en siete de cada 10 mexicanos adultos, con lo que México está considerado como el país con el primer lugar en consumo de refrescos, con 163 litros al año por persona, incluso $40 \%$ más que lo que se consume en los Estados Unidos, donde consumen, en promedio, 118 litros $^{(31)}$. Así, desde hace varias décadas en México, los refrescos se han convertido en elementos clave de la cultura alimentaria mexicana, asociándose a situaciones festivas y de placer que se reproducen cotidianamente en las comidas familiares $^{(32)}$ y esta alta ingesta de bebidas azucaradas genera que los mexicanos sean los más obesos y quienes más mueren de diabetes en América Latina ${ }^{(32)}$.

En contraste, culturas como la japonesa, que tiene población con muy bajas tasas de obesidad, más longevidad y estilos de vida más sanos, se consume más cantidad de agua sola ${ }^{(29)}$. Un estudio realizado en Hiroshima, en 305 adultos japoneses de entre 22 y 77 años, se encontró un IMC de 15,4 a $35,0 \mathrm{~kg} / \mathrm{m}^{2}$; el $58 \%$ de los encuestados comenzaban sus comidas con un plato de verduras y el $74 \%$ de los participantes comían en un tiempo promedio de 10 a 29 min; aquellos que se saltaban el desayuno (19\%) tenían un IMC significativamente más alto que aquellos que desayunaban. Quienes dejaron de comer con un $80 \%$ de saciedad tenían un IMC y niveles de presión arterial más bajos en comparación con los que comieron hasta un $100 \%$ de saciedad ${ }^{(29)}$, lo cual no ocurre en México, ya que sentirse satisfecho puede ser más importante que comer sano, por lo tanto, se prefieren los alimentos densos en energía de bajo precio para alcanzar la saciedad.

Otra cuestión es el tema del sabor, ya que se identifica que quienes tienen IMC alto, sobrevaloran disfrutar de alimentos muy sabrosos en comparación con personas de peso normal ${ }^{(32)}$, siendo una de las diferencias más preocupantes, el bajo consumo de agua en los mexicanos y exceso de bebidas azucaradas y refresco de cola ${ }^{(31,33)}$. Por lo anterior, y como fenómeno social que comúnmente se observa de forma individual, se recomienda incluir elementos culturales en estudios sobre alimentación y obesidad, ya que los alimentos no solo nutren, sino que expresan diversidad cultural, pues existen sociedades "obesígenas"(34) que requieren aproximaciones ecológicas, sistémicas, grupales, familiares y comunitarias.

Desde otra perspectiva, el contar con mayor nivel educativo no asegura hábitos de alimentación y peso saludables, aunque autores como SagarraRomero et al. ${ }^{(35)}$ señalan que el nivel educativo está inversamente relacionado con la composición corporal, pero solo en jóvenes y adultos, no así en adultos mayores. Esto orienta a considerar la etapa de desarrollo madurativo como parte del problema, pues este autor encontró en datos de 2.706 adultos mayores españoles, que un bajo nivel de estudios aumenta las posibilidades de padecer sobrepeso y obesidad en las mujeres y de tener mayor perímetro de cintura en ambos sexos de adultos mayores ${ }^{(35)}$.

Por otra parte, un estudio en población española de adultos jóvenes universitarios ${ }^{(36)}$ señala que los hábitos de alimentación se caracterizan por alto consumo de alimentos cárnicos y lácteos, reflejado en el exceso de grasa saturada, colesterol, proteína animal y baja ingesta de frutas y verduras; aun así esa población presentaba alta proporción de normopeso y hábitos alimentarios adecuados respecto a la distribución y número de comidas (4 al día), mejor a otras poblaciones similares estudiadas. Una de las principales diferencias con los mexicanos se encuentra en el alto consumo de hidratos de carbono, aunque en ambas poblaciones hay bajo consumo de vegetales ${ }^{(36)}$.

Contrario a México, en jóvenes estudiantes ecuatorianos, la prevalencia de obesidad y sobrepeso es de $17,1 \%$ (vs 35\%) ${ }^{(8,37)}$, aunque coincide que los jóvenes con sobrepeso u obesidad tienen hábitos alimenticios inapropiados, con alto consumo de dulces, bollería y embutidos, $22 \%$ de jóvenes delgados no desayunan y $14 \%$ de ellos siguen dietas hipocalóricas ${ }^{(37)}$. Al igual que en México, los jóvenes realizan estrategias insanas para el control del peso y presentan patrones dietéticos "occidentalizados" poco saludables ${ }^{(24,23,25)}$. En las poblaciones de jóvenes ecuatorianos, mexicanos y españoles, se informa consumo de alcohol, práctica 
de actividad física menor en los jóvenes obesos, disminución de frutas, verduras, pescado y aumento de comida procesada ${ }^{(23,24,25,37)}$.

En las familias, destacan rituales en las comidas, festejos, reuniones de fines de semana para comer antojitos y en general alimentos altos en grasa, azúcares y harinas refinadas, así como bebidas azucaradas y bebidas con alcohol, sin ninguna restricción. Se observa la práctica inadecuada de consumo de alimentos al ver la televisión, o el uso del teléfono celular mientras comen, entre otros hábitos, así como el comer en restaurantes de comida rápida o en tiendas de conveniencia, cuya característica es la comida rápida y de bajo costo, donde se consumen productos procesados o refrigerados, sólo para cubrir la necesidad de comer, dejando a un lado el valor nutricional de los $\operatorname{alimentos}^{(9)}$.

Algunas de las limitaciones de este estudio fue la búsqueda de artículos en idiomas distintos al inglés y español, la no inclusión de artículos con restricciones de acceso o de distintos tipos de obras o producción académica. Sin embargo, los resultados pueden ser de interés y utilidad para poblaciones similares a la mexicana, tanto para nuevas investigaciones, como para intervenciones a nivel asistencial.

Los hallazgos sugieren estrategias integrales y holísticas, que consideren elementos como los identificados en esta revisión, con enfoque preventivo. Es necesario realizar indagaciones con

\section{REFERENCIAS}

1. Organización Mundial de la Salud (OMS). Obesidad y sobrepeso, datos y cifras [Internet]. 2020 [citado 19 abr 2021]. Disponible en: https:// www.who.int/es/news-room/fact-sheets/detail/ obesity-and-overweight

2. Dávila-Torres J, González-Izquierdo J, BarreraCruz A. Panorama de la obesidad en México. Rev Med Inst Mex Seguro Soc [Internet]. 2015 [citado 20 ago 2019]; 53(2): 240-249. Disponible en: https://www.medigraphic.com/cgi-bin/new/ resumen.cgi? IDARTICULO $=56578$

3. ISSSTE. México ocupa el primer lugar en obesidad en Latinoamérica: ISSSTE [Internet]. 2019 [citado diseños controlados, bajo posturas teóricas de enfermería que ofrecen gran variedad de enfoques de autocuidado y cambios de comportamiento, desde donde se puedan generar instrumentos de medición para uso poblacional a nivel comunitario, clínico y preventivo, que incluyan las cuatro categorías derivadas de la revisión.

\section{CONCLUSIONES}

Las zonas rurales reportan mayor consumo de frutas, verduras, cereales y vitaminas, así como menor consumo calórico; en zonas urbanas se consume mayor cantidad de grasa, alimentos procesados y azúcares refinadas, así como menos consumo de vegetales, frutas y cereales. Pese a ello, el problema del sobrepeso y obesidad es similar en ambas zonas.

Los hombres consumen más carne, grasa y alcohol, las mujeres consumen más hidratos de carbono y azúcares refinadas, se saltan comidas y asocian el comer a las emociones.

El problema de la obesidad es mayor en mujeres que en hombres. Un mayor estrato socioeconómico sugiere una alimentación más adecuada, con menos calorías y comidas más naturales, así como mayor ejercicio físico programado.

La cultura juega un papel importante en las costumbres y creencias. El nivel educativo no asegura prácticas alimentarias saludables.
19 abr 2020]; Disponible en: https://www.gob.mx/ issste/prensa/mexico-ocupa-el-primer-lugar-enobesidad-en-latinoamerica-issste?idiom=es

4. Instituto Nacional de Salud Pública (INSP). La obesidad en México. Estado de la política pública y recomendaciones para su prevención y control [Internet]. $1^{\text {a }}$ ed. 2018 [citado 20 oct 2019]; 1(1): 31-41. Disponible en: https://www.insp.mx/ produccion-editorial/novedades-editoriales/4971obesidad-mexico-politica-publica-prevencioncontrol.html

5. Ceballos-Macías JJ, Pérez N-JR, Flores-Real JA, Vargas-Sánchez J, Ortega-Gutiérrez G, MadrizPrado R. et al. Obesidad. Pandemia del siglo XXI. Rev. Sanid. Mil. [Internet]. 2018 [citado 21 oct 
2019]; 72(5-6): 332-338. Disponible en: http:// www.scielo.org.mx/pdf/rsm/v72n5-6/0301-696Xrsm-72-5-6-332.pdf

6. Rubio-Herrera MA, Bretón-Lesmes I. Obesity in the COVID era: A global health challenge. Endocrinol Diabetes Nutr [Internet]. 2021 Feb [citado 20 abr 2021]; 68(2): 123-129. Disponible en: https://www.ncbi.nlm.nih.gov/pmc/articles/ PMC7577699/

7. Tenorio-Mucha J, Hurtado-Roca Y. Revisión sobre obesidad como factor de riesgo para mortalidad por COVID-19. Acta méd Perú [Internet]. 2020 Jul [citado 20 abr 2021]; 37(3):324-329. Disponible en: 1728-5917-amp-37-03-324.pdf (scielo.org.pe)

8. Secretaría de Salud. Encuesta Nacional de Salud y Nutrición (ENSANUT) [Internet]. 2018 [citado 20 oct 2019]. Disponible en: https://ensanut. insp.mx/encuestas/ensanut2018/doctos/informes/ ensanut_2018_presentacion_resultados.pdf

9. Martínez I, Villezca P. La alimentación en México. Un estudio a partir de la encuesta nacional de ingresos y gastos de los hogares y de las hojas de balance alimenticio de la FAO. Ciencia UANL [Internet]. 2005 [citado 19 oct 2019]; 8(1): 196208. Disponible en: https://www.redalyc.org/ pdf/402/40280207.pdf

10. Gómez DY, Velázquez REB. Salud y Cultura Alimentaria en México. Revista Digital Universitaria (RDU) [Internet]. 2019 [citado $19 \mathrm{abr}$ 2021]; 20(1): 1-12. Disponible en: http://doi. org/10.22201/codeic.16076079e.2019.v20n1.a6

11. Aranda PS. Alimentación en México. Cuicuilco [Internet]. 2014 [citado 19 abr 2021]; 21(61): 373-378. Disponible en: http://www.scielo.org. $\mathrm{mx} / \mathrm{pdf} /$ cuicui/v21n61/v21n61a19.pdf

12. Martínez E. ¿Conocemos lo que comemos? Una perspectiva nutricional. Nutr Hosp [Internet]. 2018 [citado 10 oct 2019]; 35 (4): 61-65. Disponible en: http://dx.doi.org/10.20960/nh.2128

13. Torres J, Contreras S, Lippi L, Huaiquimilla M, Leal R. Hábitos de vida saludable como indicador de desarrollo personal y social: discursos y prácticas en escuelas. Calidad en la educación [Internet]. 2019 [citado 10 abr 2021]; (50): 357-392. Disponible en: https://dx.doi.org/10.31619/caledu.n50.728

14. Crossetti M. Revisión integrativa de la investigación en enfermería, el rigor científico que se le exige. Rev Gaúcha Enferm [Internet]. 2012 [citado 10 oct 2019]. 33(2): 10-11. Disponible en: https://www. scielo.br/pdf/rgenf/v33n2/02.pdf

15. Navarro-Meza M, Martínez A, López-Espinoza A, López-Uriarte P, Benavides M. Comparación en la ingesta de alimentos de adultos que residen en una zona rural y urbana de Jalisco, México. Revista Mexicana de Trastornos Alimentarios
[Internet]. 2014 [citado 28 oct 2019]; 5: 1119. Disponible en: https://www.redalyc.org/ pdf/4257/425741621003.pdf

16. Caamaño M, Ronquillo D, Kimoto R, García O, Long K, Rosado J. Beliefs and motives related to eating and body size: a comparison of high-BMI and normal-weight young adult women from rural and urban areas in México. 2016; BMC Public Health [Internet]. 2020 [citado 29 mar 2020]; 16(1014): 1-12. Disponible en: https:// bmcpublichealth.biomedcentral.com/track/ pdf/10.1186/s12889-016-3695-4.pdf

17. Marín A, Sánchez G, Maza L. Prevalencia de obesidad y hábitos alimentarios desde el enfoque de género: el caso de Dzutóh, Yucatán, México. Estudios Sociales [Internet]. 2014 [citado 29 oct 2019]; 22 (44): 64-90. Disponible en: http://www. scielo.org.mx/pdf/estsoc/v22n44/v22n44a3.pdf

18. Dosamantes-Carrasco L, Méndez-Hernández P, Flores Y, Siani C, Denova-Gutiérrez E, GallegosCarrillo K, et al. Influence of mealtime habits on the risk of weight gain and obesity in Mexican adults. Public Health Nutrition [Internet]. 2020 [citado 25 mar 2020]; 20(2): 220-232. Disponible en: 10.1017 / S1368980016002184

19. Rivera JA, Pedraza LS, Aburto TC, Batis C, Sánchez-Pimienta TG, González DCT, et al. Overview of the Dietary Intakes of the Mexican Population: Results from the National Health and Nutrition Survey 2012. J Nutr [Internet]. 2016 [citado 8 oct 2019]; 146(9): 1851S-5S. Disponible en: https://doi.org/10.3945/jn.115.221275

20. Gaona-Pineda E, Martínez-Tapia B, ArangoAnguita A, Valenzuela-Bravo D, Gómez-Acosta L, Shamah-Levy T, et al. Consumo de grupos de alimentos y factores sociodemográficos en población mexicana. Salud Pública de México [Internet]. 2018 [citado 28 oct 2019]; 60(3): 272282. Disponible en: https://www.saludpublica.mx/ index.php/spm/article/view/8803/11500

21. Barquera $S$, Campos-Nonato I, Hernández-Barrera L, Pedroza A, Rivera-Dommarco J. Prevalencia de obesidad en adultos mexicanos 2000-2012. Salud pública Méx [Internet]. 2013 [citado 20 oct 2019]; 55(supl. 2): 151-160. http://www.scielo.org.mx/ pdf/spm/v55s2/v55s2a12.pdf

22. Reséndiz E, Aguilera P, Rocher M. Estilo de vida e índice de masa corporal de una población de adultos del sur de Tamaulipas, México. Aquichan [Internet]. 2010 [citado 28 oct 2019]; 10(3): 244252. Disponible en: http://www.scielo.org.co/pdf/ aqui/v10n3/v10n3a06.pdf

23. Gómez CZ, Landeros RP, Romero VE, Troyo SR. Estilos de vida y riesgos para la salud en una población universitaria. Revista de Salud 
Pública y Nutrición [Internet]. 2016 [citado 9 oct 2019]; 15(2): 9-14. Disponible en: https:// www.medigraphic.com/cgi-bin/new/resumen. cgi? IDARTICULO=67869

24. Trujillo-Hernández B, Vásquez C, AlmanzaSilva J, Jaramillo-Virgen M, Mellin-Landa T, Valle-Figueroa $\mathrm{O}$, et al. Frecuencia y factores de riesgo asociados a sobrepeso y obesidad en universitarios de Colima, México. Revista de Salud Pública [Internet]. 2010 [citado 26 oct 2019]; 12(2): 197-207. Disponible en: https://revistas. unal.edu.co/index.php/revsaludpublica/article/ view/33150/33162

25. Betancourt-Núñez A, Márquez-Sandoval F, González-Zapata L, Babio N, Vizmanos B. Unhealthy dietary patterns among healthcare professionals and students in México. BMC Public Health [Internet]. 2018 [citado 28 oct 2020]; 18(1246): 1-14. Disponible en: https:// bmcpublichealth.biomedcentral.com/track/ pdf/10.1186/s12889-018-6153-7.pdf

26. Colunga LL. Obesidad y sedentarismo en poblaciones rural y urbana (Tesis de Grado, Maestría en Ciencias de Enfermería). Facultad de Enfermería, Universidad Autónoma de Nuevo León. México [Internet]; 2005 [citado 9 abr 2021]. Disponible en: http://eprints.uanl. $\mathrm{mx} / 1632 / 1 / 1080126956$.PDF

27. López DL, Torre M, Bellido D, Monereo S, Lecube A, Sánchez E, Tinahones FJ. Ganancia de peso durante el confinamiento por la COVID-19; encuesta de la Sociedad Española de Obesidad. bmi journal [Internet]. 2020 [citado 16 abr 2021]; 10: 2774-2781. Disponible en: https://www.bmijournal.com/index.php/bmi/article/view/739

28. Díaz-Castrillón F, Cruzat-Mandich C, OdaMontecinos C, Inostroza M, Saravia S, Lecaros J. Comparación de mujeres jóvenes con obesidad y normopeso: vivencia corporal, hábitos saludables y regulación emocional. Rev chil nutr [Internet]. 2019 Jun [citado 16 abr 2021]; 46(3): 308-318. Disponible en: https://scielo.conicyt.cl/pdf/rchnut/ v46n3/0717-7518-rchnut-46-03-0308.pdf

29. Toh YEW, Hirohiko M. Japanese dietary habits: Results from a questionnaire survey on 305 health check-up participants. Clin Health Promot [Internet]. 2017 [citado 20 nov 2019]; 7(1): 12-17. Disponible en: http://clinhp.org/ifile/Vol7_Iss1_ p12_p17.pdf

30. Matus NP, Álvarez GC, Nazar DA, Mondragón R. Percepciones de adultos con sobrepeso y obe- sidad y su influencia en el control de peso en San Cristóbal de Las Casas, Chiapas. Estud soc [Internet]. 2016 [citado 26 sep 2019]; 24-25(47): 380-409. Disponible en: 0188-http://www.scielo. org. mx/pdf/estsoc/v24-25n47/0188-4557estsoc-24-47-00380.pdf

31. Delgado S. México, primer consumidor de refrescos en el mundo [Internet]. 2019 [citado 23 mar 2021]. Disponible en: https://www.gaceta. unam.mx/mexico-primer-consumidor/

32. Pedraza DF. Obesidad y pobreza: marco conceptual para su análisis en Latinoamérica. Saude e soc [Internet]. 2009 [citado 21 abr 2021]; 18(1): 103117. Disponible en: http://dx.doi.org/10.1590/ S0104-12902009000100011.

33. Cabrera E. El alto consumo de refrescos en México es resultado del entramado de procesos económicos, políticos y culturales. Resonancias (Blog Instituto de Investigaciones Sociales de la UNAM) [Internet]. 2019 [citado 8 dic 2020]. Disponible en: https:// www.iis.unam.mx/blog/el-alto-consumo-derefrescos-en-mexico-es-resultado-del-entramadode-procesos-economicos-politicos-y-culturales/

34. Pérez Gil RS, Romero JG. Imagen corporal en mujeres rurales de la Sierra Juárez y la costa de Oaxaca: una aproximación nutrio-antropo-lógica. Estudios Sociales [Internet]. 2008 [citado $22 \mathrm{abr}$ 2021]; 16 (32): 80-111. Disponible en: https:// www.redalyc.org/articulo.oa?id=41703203

35. Sagarra-Romero L, Gómez-Cabello A, PedreroChamizo R, Vila-Maldonado S, Gusi-Fuertes N, Villa-Vicente JG, et al. Relación entre el nivel educativo y la composición corporal en personas mayores no institucionalizadas: Proyecto Multicéntrico EXERNET. Rev Esp Salud Pública [Internet]. 2017 [citado 7 abr 2021]; 91: e201710041. Disponible en: https://scielo.isciii.es/ pdf/resp/v91/1135-5727-resp-91-e201710041.pdf

36. Márquez MR, Beato VP, Tormo GM. Hábitos de vida, de alimentación y evaluación nutricional en personal sanitario del hospital de Mérida. Nutr Hosp [Internet]. 2015 Abr [citado 22 abr 2021]; 31(4): 1763-1770. Disponible en: http://dx.doi. org/10.3305/nh.2015.31.4.8455.

37. Borjas SM, Loaiza DL, Vásquez PR, Campoverde PP, Arias CK, Chávez CE. Obesidad, hábitos alimenticios y actividad física en alumnos de educación secundaria. Rev Cubana Invest Bioméd [Internet]. 2017 [citado 21 abr 2021]; 36(3). Disponible en: http://scielo.sld.cu/pdf/ibi/v36n3/ ibi08317.pdf 